\title{
Radical Vaginal Hysterectomy and Trachelectomy in Early-Stage Cervical Cancer
}

\author{
Erasmo Bravo ${ }^{1,2,3^{*}}$, Catalina Alonso Montemurro1, Sergio Rojas Sepulveda ${ }^{1,3}$, \\ Jaime Cartagena Bustamante ${ }^{1,3}$, Hector Pinto ${ }^{2,3}$ \\ ${ }^{1}$ Oncologic Gynecology Unit, Gustavo Fricke Hospital, Viña del Mar, Chile \\ ${ }^{2}$ Oncologic Gynecology Unit, Van Buren Hospital, Valparaíso, Chile \\ ${ }^{3}$ Department of Gynecology and Obstetrics, University of Valparaíso, Valparaíso, Chile \\ Email: ${ }^{*}$ erasmobravo@gmail.com
}

Received 8 April 2014; revised 5 May 2014; accepted 13 May 2014

Copyright (C) 2014 by authors and Scientific Research Publishing Inc.

This work is licensed under the Creative Commons Attribution International License (CC BY).

http://creativecommons.org/licenses/by/4.0/

(c) (i) Open Access

\section{Abstract}

Objective: To communicate a minimally invasive technique for surgical handling of early-stage cervical cancer and its results. Methods: 110 patients with cervical cancer in stages IA2 and IB1, all of them operated in a period of 5 years in both hospitals, are presented. Laparoscopic systemic pelvic lymphadenectomy with radical vaginal hysterectomy or radical vaginal trachelectomy was performed to patients, with the exception of those patients who had compromised nodes detected in contemporary biopsy. Results: Between April 2008 and May 2013, 110 patients were submitted to this technique. 15 patients had their surgery aborted: 13 presented positive nodes for carcinoma in contemporary biopsy and 2 had extensive cervical compromise when performing radical vaginal hysterectomy or trachelectomy. Analysis of the remaining 95 cases shows an average age of 43.9 years (26 - 61), all of them had given birth before, $23(21 \%)$ of them through C-section. BMI averaged 30.5 and $31(28.2 \%)$ had cone surgery performed previously. Average duration of surgery was of 220 minutes. Postoperative hospitalization averaged 3.1 days. Bleeding volume was estimated at $125 \mathrm{cc}$ and one patient required blood transfusion. En 25 patients' uterine annexes were kept and all of them were suspended by means of laparoscopy. On average, 25.4 pelvic nodes were obtained. Complications $13.6 \%$ with eight patients suffered bladder injury, two had rectovaginal fistula, 3 patients requires surgical repair of ureteral obstruction, two patients present thromboembolic disease. The disease-free and overall survivals are consistent with reports in the literature. Conclusion: We believe that handling patients with this technique is possible and has the advantages of vaginal and laparoscopic surgery with minimal complications.

\section{Keywords}

Radical Vaginal Hysterectomy, Radical Vaginal Trachelectomy, Early-Stage Cervical Cancer

\footnotetext{
${ }^{*}$ Corresponding author.
} 


\section{Introduction}

Radical Vaginal Hysterectomy (RVH) was first described by surgeon Anton Pawlik and it was made common in cervical cancer by Frederik Schauta at the beginning of the twentieth century, obtaining a descent in mortality rates compared to Wertheim's abdominal technique. Schauta's technique was later modified by Amreich and Stockel [1]. When Meigs introduced lymphadenectomy with Radical Abdominal Hysterectomy [RAH], Schauta's operation was virtually forgotten, given the impossibility of nodes removal through the vagina [2]. With the advent of laparoscopic lymphadenectomy in the 1980s with Dargent, Querleu and Childers, RVH has been reborn and it has progressively become more common in several centers worldwide [3]-[5].

RAH is a surgical procedure which has significant morbility with intestinal and bladder dysfunction and slow recovery. The advantages of laparoscopic surgery are widely known, allowing fast recovery, less postoperative pain, absence of laparotomy and its complications and, technically, it has a better view of pelvic and abdominal anatomical structures. On the other hand, vaginal hysterectomy also has advantages compared to an abdominal one: with a better and faster recovery, less complications, shorter postoperative hospitalization, it's less expensive and leaves no visible scar.

RVH assisted by videolaparoscopy merges both techniques, obtaining the advantages of both in a single patient and in an important surgery in oncologic gynecology.

In 1994, Dr. Daniel Dargent described the technique of radical vaginal trachelectomy (RVT), which removes the cervix, the parametria, and the vaginal cuff, in conjunction with a systematic laparoscopic pelvic lymphadenectomy, preserving the corpus uteri and its pedicles, as a way of preserving fertility in those young patients who have not completed their parity.

The present document intends to show the preliminary experience with this technique in the surgical treatment of early-stage cervical cancer within our units.

\section{Methods}

In April 2008, there was consecutive recruitment in a prospective and protocolized way a cohort of patients carrying early-stage cervical cancer (IA2, IB1, IIA1), which were then submitted to RVH or RVT, with sistematic pelvic lymphadenectomy by videolaparoscopy, in the Oncologic Gynecology Units in Gustavo Fricke and Carlos Van Buren Hospitals in Viña del Mar and Valparaíso, respectively. Patients were diagnosed in the Cervical Pathology clinic of their respective units and clinically assessed the degree of progression of the cancer in a meeting of the Institution's Oncogynecologic committee. All patients are subjected to axial computed tomography. Afterward, their surgery was planned within the time frames established by the GES Clinical Guide for this pathology. In this report, certain demographic characteristics are analyzed, like age, parity, body-mass index, previous surgical records; and surgical results like duration of laparoscopic and vaginal surgery, estimated bleeding, use of transfusion, conservation of uterine annexes, intra- and postoperative complications, duration of hospitalization, overall survival and disease-free survival. Anatopathological data, like clinical stage, tumor size, node count and hystological type, was also reported. Finally, the need of radiochemotherapy is mentioned.

\section{Technique}

With general anesthesia and in position for lythotomy, laparoscopic surgery is started, with a $10 \mathrm{~mm}$ umbilical open entry and 3 suprapubic punctures, the middle one of $10 \mathrm{~mm}$ and the lateral ones measuring $5 \mathrm{~mm}$. With 15 mmHg of pneumoperitoneum the abdominal cavity and pelvis is explored, separating adherences when needed. Depending on the patient's age, uterine annexes are kept or not, which are then coagulated with bipolar and the uteroovarian or infundibulopelvic ligaments are cut with scissors. In patients where ovaries are kept, these are stuck to the abdominal wall above the promontory also by endoscopic means. Afterward, lymphadenectomy is started removing all of the nodular tissue of the common, external and internal iliac artery and the obturator foramen above and below the obturator nerve, on both sides. Nodes are extracted with the sac through the navel and also through here the uterine artery is tied on its exit level to the hypogastric artery using metallic clips.

On a second stage the surgery is switched to the vaginal path and the extension of the vaginal cuff is demarcated. With a cold scalpel or an electroscalpel a circular incision is made in the vagina and it is dissected until the cervix is covered with it, grabbing it with Chroback forceps. The vagina is released introducing a Deaver separator in the vesicouterine space, without opening the peritoneum. A paravesical space is opened, which ex- 
poses the vesical pillar. At this level it is possible to localize the ureter by touch, which allows its dissection and subsequent displacement to the cephalic area, which in turn permits the cutting of the vesical pillar near the bladder and ureter. Afterward, the back end of the vaginal sac is opened and the pararectal area is entered with scissors, with the uterosacral ligament widely exposed because of this, which is cut as close to the rectum as possible. With it, the uterus is united to the pelvic wall through the paracervix, which is cut and tied to the former as close as it can. Finally the uterus and the parametrial tissues are tractioned and extracted through the vagina. Hemostasis is completed and the vaginal dome is closed with a polyglycolic acid suture. Hemostasis is checked laparoscopically and drainage is left which is removed through any of the $5 \mathrm{~mm}$ punctures. A vesical catheter is installed. Punctions are closed and with it, the surgery is completed. On the first postoperative day the vesical catheter is removed and postmictional catheterism is indicated, which is suspended when the residue is under 100 cc.

In vaginal radical trachelectomy, the same technique is used, but the difference is cervical branch of the uterine artery is linked followed by the division of the cervix at the level of its attachment with the uterine isthmus. A rapid biopsy of the edge of the upper section is performed to ensure a free margin of at least $5 \mathrm{~mm}$ and a Shirodkar cerclage is set in place. Finally, vaginal closure is carried out around the isthmus with laparoscopic confirmation of hemostasis.

\section{Results}

Between April 2008 and May 2013, 110 patients have surgery with the described technique. In 15 patients surgery is aborted due to findings during the procedure, 13 of them because of nodes positive for neoplasia in lymphadenectomy and the other two because of extensive cervical compromise with cancer during the initial moments of the vaginal stage. In all of them with positives pelvic nodes, we did a laparoscopic lumbar aortic nodular sampling, and are submitted to radiochemotherapy.

In the remaining 95 patients, 10 underwent vaginal radical trachelectomy with laparoscopic pelvic lymphadenectomy, and the remaining 85 underwent vaginal radical hysterectomy with laparoscopic pelvic lymphadenectomy. The age range was 19 to 84, the average was 43.1. All of them had given birth before, with 23 (21\%) of them having done so by means of a C-section. BMI averaged 30.5 with a range going from 23.66 to 43.05 .31 (28.2\%) patients had been previously submitted to conization. Average duration of surgery was of 220 minutes, being 180 and 300 minutes the shortest and longest duration, respectively. Postoperative hospitalization averaged 3.1 days, with the shortest hospitalization being 1 day, and the longest being 17, which was due to the patient having severe dysfunction of the urinary tract. 75 (79\%) patients remain hospitalized for 2 or 3 days, and the remaining 20 for more than 3 days. Bleeding volume was estimated at $125 \mathrm{cc}$ and one patient required blood transfusion. No patients need reoperation for postoperative hemorrhage. Uterine annexes are kept in 25 patients (26.3\%) and all of them are suspended by means of laparoscopy. On average, 25.4 nodes are obtained (with a minimum of 9 and a maximum of 60). Ninety patients are classified as being in stage $1 B 1$ and 5 as 1A2. In 62 patients (62\%), the hystological type was that of squamous cell carcinoma, 2 adenosquamous carcinoma and the rest were adenocarcinoma.

Thirty one patients (28.2\%) require postoperative radiochemotherapy due to risk of recurrence, including 15 patients whose surgery was aborted in the intraoperative period. Fifteen patients present complications (13.6\%), 8 patients suffer vesical lesions (2 during the laparoscopic lymphadenectomy and 6 during the vaginal procedure, all of which were repaired immediately). Two patiens had rectovaginal fistula and 3 patients requires surgical repair of ureteral obstruction. Two patients present thromboembolic disease with good evolution.

Of patients undergoing radical vaginal trachelectomys, one of them, the intervention was performed in a patient with a pregnancy of 11 weeks, with good evolution and cesarean delivery at 37 weeks. Another patient completed her second pregnancy successfully after the first with poor outcome of prematurity.

Statistical analysis: Survival curves were calculated using the Kaplan-Meier method.

The disease-free survival at 3 years is 95\% with a median follow up of 21 months (Figure 1). The overall survival is too 95\% at 3 years (Figure 2) and the Figure 3 shows disease-free survival in relation to histological type.

\section{Discussion}

Usually, radical hysterectomy has been done through the abdomen since pelvic lymphadenectomy was intro- 
Survival Function

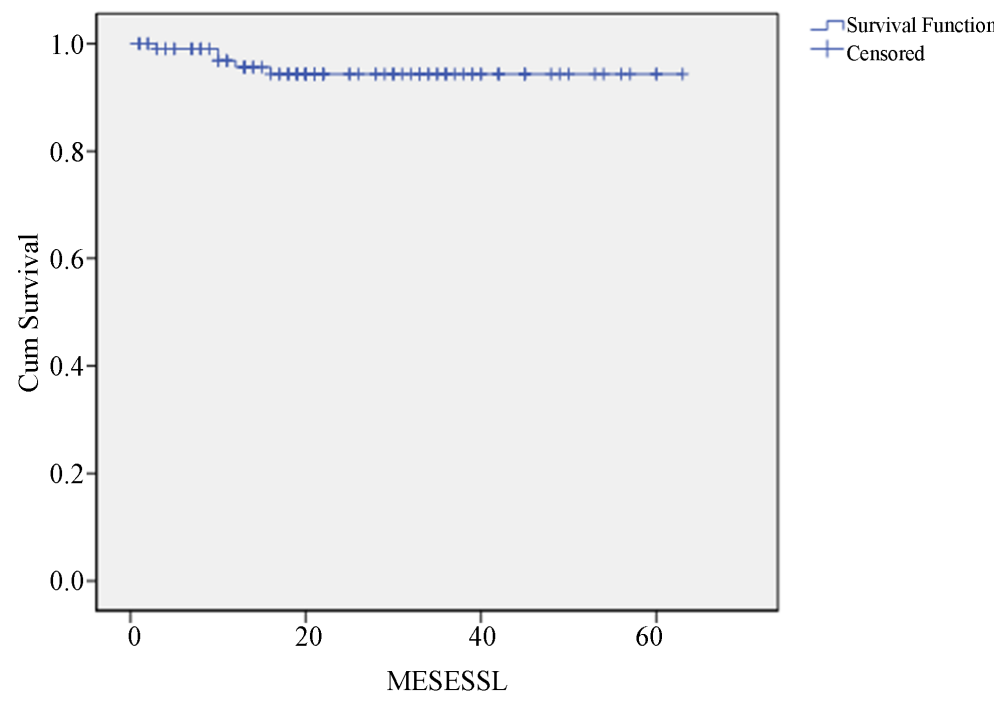

Figure 1. Disease free survival of the cohort, 5 events in interval, end point July 2013. Follow up median 21 months. DFS 3 years, 95\%.

Survival Function

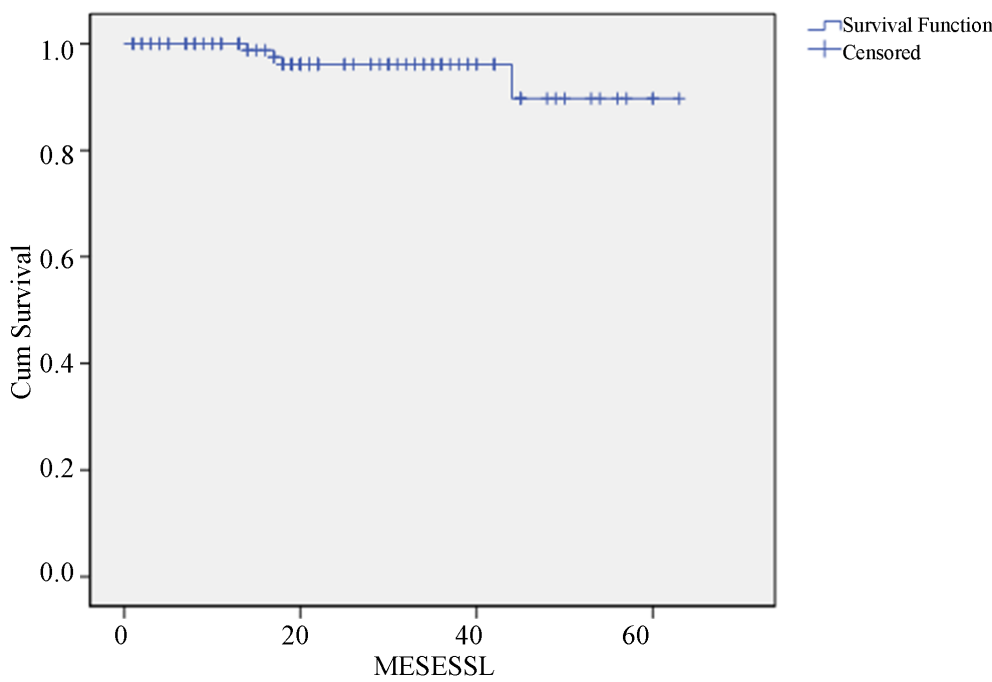

Figure 2. Overall survival of the cohort, 4 deaths in interval, end point July 2013 follow up median 21 months. OS 3 years, 95\%.

duced as part of the procedure. This way, Schauta's radical hysterectomy stopped being in use despite fewer number of complications. Nowadays, several centers worldwide have developed the vaginal technique since the introduction of laparoscopic pelvic lymphadenectomy at the end of the 1980s and beginning of the 1990s, with the benefits provided by laparoscopic and vaginal surgery. The present institutions' gynecological services have a remarkable trajectory in the former techniques, with 15 years' worth of experience. With that in mind, in 2008 the researchers decided to deliver this experience to both hospitals' Oncologic Gynecology. This research shows the results obtained with this procedure in the first 110 consecutive patients that had early-stage cervical cancer, submitting them to laparoscopic systemic pelvic lymphadenectomy and afterwards to radical vaginal hysterectomy or trachelectomy in those patients who wish to preserve their fertility. In 2007, Sonoda [1] does a detailed description of the surgical technique similar to the one done by the current team. In contrast to Schauta's proce- 
Survival Functions

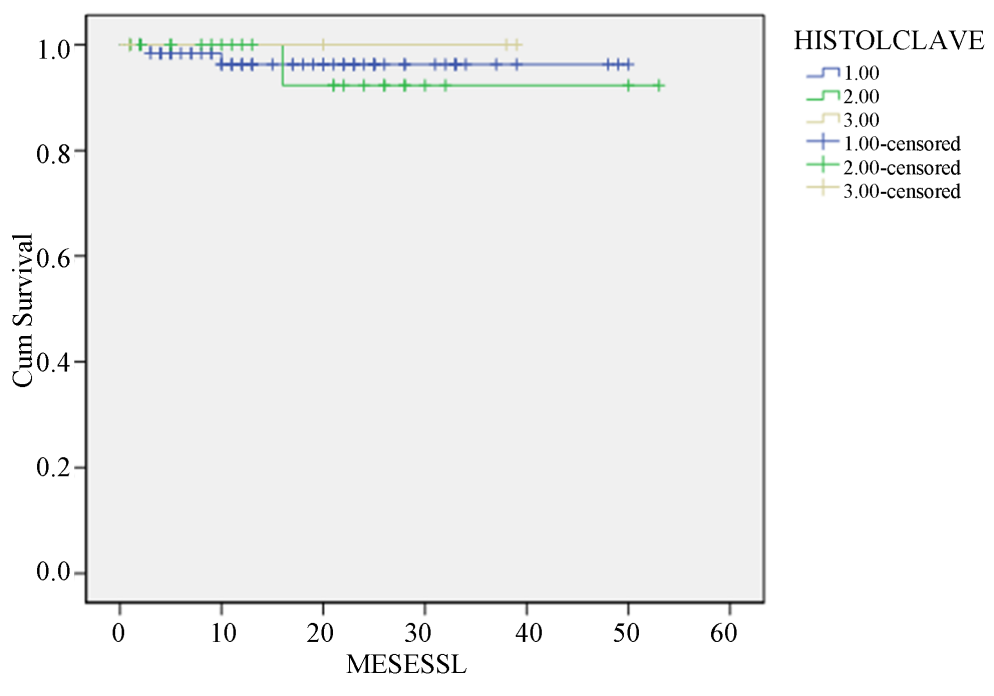

Figure 3. Disease free survival of the cohort for histology, end point July 2013, 1 escamous carcinoma; 2 adenocarcinoma; 3 adenoescamous carcinoma. Follow up median 21 months. P Ns.

dure, Schuharts's incision is not done because it is considered extensive and unnecessary. In 2003, Hertel [6] publishes 200 cases of RVH assisted by videolaparoscopy and, with 40 months of follow-up, obtains a projected $83 \%$ survival rate of 5 years. Therefore, it's concluded that RVH is an adequate technique for early-stage cervical cancer. Morgan [7], in 2007, compares vaginal and abdominal techniques in the first 30 cases and concludes that both are similar in terms of recurrence and complications after 31 months of follow-up. In January, 2010, Pahisa [8], from Barcelona, Spain, publishes the experience of 11 years with 67 patients submitted to this technique and compares it with a historical series of 23 cases operated with Wertheim-Meigs' technique. It is concluded that the vaginal procedure is safe, has a faster postoperative recovery and survival rate is similar in both procedures.

In the series of this study, of 110 cases, 95 completed the surgery. The other 15, abort their surgery for pelvic lymph node metastatic tumor (13 patients) or major commitment to initiate proceedings vaginal (2 patients). Ten patients undergoing trachelectomy, one with 11 weeks gestation, published during 2012 in Ginecologic Oncology Case Reports [9]. The average duration of 220 minutes is lower than Pahisa's 260. Patients' average BMI of $30 \mathrm{~kg} / \mathrm{m}^{2}$ exceeds the spanish patients' average of $25 \mathrm{~kg} / \mathrm{m}^{2}$, which offers more technical difficulty and demonstrates a lesser selection of the first series of patients. Node count is clearly superior in this study (with an average of 25.4), compared to Pahisa's (15.2 nodes). Only one patient of this study requires blood transfusion, while the spanish study required transfusions for $15 \%$ of its patients. Amount of complications are similar, in the sense that there was an absence of ureteral or intestinal lesions in both studies. Finally, the patients in this study had a postoperative hospitalization of 3.1 days on average, which is less than Pahisa's average of 4.9 days.

The complication rate (13.6\%) is consistent with the reported in the literature, highlighting intraoperatoria bladder injuries (8 patients) as part of the learning curve, they are presented at the beginning of the experience.

The overall and disease-free survival of this group of patients is consistent with reports in the literature.

It is concluded that the handling of this group of patients, presenting early-stage cervical cancer is possible, with oncological safety and obtaining the benefits of vaginal and laparoscopic surgery. On the other hand, the learning of this technique is a necessary step in order to be able to proceed with Radical Vaginal Trachelectomy, which allows preserving the fertility in the patient.

\section{References}

[1] Sonoda, Y. and Abu-Rustum, N. (2007) Schauta Radical Vaginal Hysterectomy. Gynecologic Oncology, 104, $20-24$. http://dx.doi.org/10.1016/j.ygyno.2006.10.026 
[2] Angioli, R., Martin, J., Heffernan, T., et al. (2001) Radical Vaginal Hysterectomy Classic and Modified. Surgical Clinics of North America, 81, 829-838.

[3] Dargent, D. (1987) A New Future for Schauta's Operation through Pre-Surgical Retroperitoneal Pelviscopy. European Journal of Gynaecological Oncology, 8, 292-296.

[4] Querleu, D., LeBlanc, E. and Castellain, B. (1991) Laparoscopic Pelvic Lymphadenectomy in the Staging of Early Cervical Carcinoma of the Cervix. American Journal of Obstetrics \& Gynecology, 164, 579-581. http://dx.doi.org/10.1016/S0002-9378(11)80025-6

[5] Childers, J.M., Hatch, K.D., Tran, A.N., et al. (1993) Laparoscopic Para-Aortic Lymphadenectomy in Gynecologic Malignancies. Obstetrics \& Gynecology, 82, 741-747.

[6] Hertel, H., Kohler, C., Michels, W., et al. (2003) Laparoscopic-Assisted Radical Vaginal Hysterectomy (LARVH): Prospective Evaluation of 200 Patients with Cervical Cancer. Gynecologic Oncology, 90, 505-511. http://dx.doi.org/10.1016/S0090-8258(03)00378-0

[7] Morgan, D., Hunter, D., McCracken, G., et al. (2007) Is laparoscopically Assisted Radical Vaginal Hysterectomy for Cervical Carcinoma Safe? A Case Control Study with Follow up. BJOG, 114, 537-542. http://dx.doi.org/10.1016/S0090-8258(03)00378-0

[8] Pahisa, J., Martinez-Román, S., Torné, A., et al. (2010) Comparative Study of Laparoscopically Assisted Radical Vaginal Hysterectomy and Open Wertheim-Meigs in Patients with Early Stage Cervical Cancer. International Journal of Gynecologycal Cancer, 20, 173-177.

[9] Bravo, E., Parry, S. and Alonso, C. (2012) Radical Vaginal Trachelectomy and Laparoscopic Pelvic Lymphadenectomy in 1B1 Cervical Cancer during Pregnancy. Gynecologic Oncology Reports, 2, 78-79. http://dx.doi.org/10.1016/j.gynor.2012.04.002 